\title{
Early seroconversion and rapidly increasing autoantibody concentrations predict prepubertal manifestation of type 1 diabetes in children at genetic risk
}

\author{
V. Parikka • K. Näntö-Salonen • M. Saarinen • \\ T. Simell • J. Ilonen • H. Hyöty • R. Veijola • M. Knip • \\ O. Simell
}

Received: 2 September 2011 / Accepted: 10 February 2012 /Published online: 23 March 2012

(C) Springer-Verlag 2012

\begin{abstract}
Aims/hypothesis The aim of the study was to investigate the timing of the appearance of autoantibodies associated with type 1 diabetes between birth and puberty, the natural fate of these autoantibodies and the predictive power of autoantibody concentrations for early progression to clinical diabetes.
\end{abstract}

Electronic supplementary material The online version of this article (doi:10.1007/s00125-012-2523-3) contains peer-reviewed but unedited supplementary material, which is available to authorised users.

V. Parikka $\cdot$ K. Näntö-Salonen · T. Simell $•$ O. Simell

Department of Pediatrics, Turku University Hospital,

Kiinamyllynkatu 4-8, PO Box 52, 20521 Turku, Finland

V. Parikka $(\bowtie) \cdot$ K. Näntö-Salonen $\cdot$ T. Simell $•$ O. Simell

Department of Pediatrics, University of Turku,

Turku, Finland

e-mail: vilpar@utu.fi

M. Saarinen

Research Centre of Applied and

Preventive Cardiovascular Medicine,

University of Turku,

Turku, Finland

J. Ilonen

Department of Clinical Microbiology,

University of Eastern Finland,

Kuopio, Finland

J. Ilonen

Immunogenetics Laboratory, University of Turku,

Turku, Finland

H. Hyöty

Department of Virology,

University of Tampere,

Tampere, Finland
Methods Children were recruited to the Type 1 Diabetes Prediction and Prevention Project, an ongoing study based on HLA-conferred genetic risk. Autoantibodies against islet cells, insulin, GAD65 and islet antigen 2 were analysed at 3-12 month intervals, starting from birth.

Results During the follow-up, 1,320 children (18.4\% of the cohort of 7,165 children) were autoantibody positive in at

\section{H. Hyöty}

Department of Clinical Microbiology,

Centre of Laboratory Medicine, Tampere University Hospital,

Tampere, Finland

R. Veijola

Department of Pediatrics, Oulu University Hospital,

Oulu, Finland

R. Veijola

Department of Pediatrics, University of Oulu,

Oulu, Finland

M. Knip

Children's Hospital, Helsinki University Central Hospital,

Helsinki, Finland

M. Knip

Institute of Clinical Medicine, University of Helsinki,

Helsinki, Finland

M. Knip

Folkhälsan Research Center,

Helsinki, Finland

M. Knip

Department of Pediatrics, Tampere University Hospital,

Tampere, Finland 
least one sample. Altogether, 184 autoantibody-positive children progressed to type 1 diabetes. Seroconversion occurred at an early age in the progressors (median 1.5 years), among whom $118(64 \%)$ and $150(82 \%)$ seroconverted to autoantibody positivity before the age of 2 and 3 years, respectively. The incidence of seroconversion peaked at 1 year of age. Compared with other autoantibody-positive children, the median autoantibody levels were already markedly higher 3 to 6 months after the seroconversion in children who later progressed to diabetes.

Conclusions/interpretation Early initiation of autoimmunity and rapid increases in autoantibody titres strongly predict progression to overt diabetes before puberty, emphasising the importance of early life events in the development of type 1 diabetes.

Keywords Autoantibodies - GADA · IA-2A · IAA · ICA . Type 1 diabetes

$\begin{array}{ll}\text { Abbreviations } \\ \text { DIPP } & \text { Type 1 Diabetes Prediction and Prevention Project } \\ \text { GADA } & \text { Autoantibody to GAD65 } \\ \text { IAA } & \text { Insulin autoantibody } \\ \text { IA-2 } & \text { Islet antigen } 2 \\ \text { IA-2A } & \text { Autoantibody to islet antigen } 2 \\ \text { ICA } & \text { Islet cell autoantibody } \\ \text { IQR } & \text { Interquartile range } \\ \text { RU } & \text { Relative units }\end{array}$

\section{Introduction}

The increase in the incidence of type 1 diabetes in most Western populations is alarming [1-3]. Data from Finland suggest that the incidence has more than tripled from 1953 to 1996 [4], reaching a rate as high as 64 per 100,000 children per year in the year 2005 [5] The incidence is increasing, especially in the youngest age group ( $0-4$ years), in whom incidence has been predicted to overtake that in the older age groups within the next 10 years $[5,6]$.

Most children who develop type 1 diabetes carry risk alleles at the HLA and other gene loci associated with susceptibility to the disease, but only a small proportion of the individuals at genetic risk ever show signs of autoimmunity or progress to clinical diabetes [7, 8]. Environmental factors are believed to interact with susceptibility genes, with mutual contributions to pathogenesis [9, 10]. The appearance of diabetes-associated autoantibodies in the serum is the first firm sign of the initiation of beta cell-specific autoimmunity, and reflects a critical increase in the risk of further progression to diabetes. Our recent data indicate that the serum metabolome may already be altered before seroconversion to autoantibody positivity occurs [11]. Yet the first signs of clinical disease only become apparent when $60-80 \%$ of the beta cells have been destroyed [12].

Our group recently studied the predictive characteristics of the presence or absence of diabetes-associated autoantibodies among children with HLA-conferred disease susceptibility in the general population [13]. Genetic risk, either associated with a family history of type 1 diabetes or with defined risk alleles, indicates, at most, a diabetes risk in the range $5-8 \%[7,14]$ by the age of 15 years, while permanent positivity for three or four diabetes-associated autoantibodies increases the risk to more than $60 \%$ over a 5 year followup [15-17]. Young age at seroconversion, positivity for multiple autoantibodies, high islet cell autoantibody (ICA) levels and persistent positivity for insulin autoantibodies (IAAs) are significant risk markers for type 1 diabetes among autoantibody-positive children [13]. Compared with recent data from the Type 1 Diabetes Prediction and Prevention Project (DIPP) [13], this analysis focuses on the role of antibodies other than ICAs, particularly IAAs. In addition, the time point of seroconversion and changes in autoantibody concentrations are studied in more detail to try to detect the earliest predictive signals of future clinical disease.

\section{Methods}

Study design and individuals In the Finnish-populationbased DIPP study, children with HLA-conferred susceptibility to type 1 diabetes have been monitored prospectively from birth. DIPP was launched in Turku in November 1994, and 1 and 3 years later in the University Hospitals of Oulu and Tampere, respectively; recruitment of newborn babies has continued since then. DIPP children born by the end of December 2007 were included in the current analysis, which uses DIPP data collected by 31 December 2008. The Ethics Committees of the participating universities and hospital districts approved the study. All families provided written informed consent for participation in the study.

Newborn infants were screened for genetic risk using cord blood, and families with a baby testing positive were invited to prospective follow-up at 3 to 12 month intervals until the age of 15 years. The Turku subcohort followed a more frequent blood-draw scheme than the other sites, as described previously $[13,18]$. DIPP children who seroconverted to positivity for any of the diabetes-associated autoantibodies analysed were transferred to a schedule with 3 month intervals. The time window for each visit was 90 days. Serum samples were drawn at each visit and analysed for ICA, IAA, autoantibody to GAD65 (GADA) and autoantibody to islet antigen 2 (IA-2A). Children who had ever had a visit interval exceeding 1 year (time window $365+45$ days) were excluded. However, if the child was 
seronegative in all samples before that interval and still seronegative in the first sample drawn after the break, a 2 year interval was allowed.

All autoantibodies in all samples from children born in or after 2003 were analysed. For the older children, ICA level alone was initially analysed. Once seroconversion to ICA positivity had occurred, all previous samples were analysed for IAA, IA-2A and GADA. The 3 to 12 month intervals between blood collections meant that the exact date of seroconversion could not be pinpointed. Thus, we defined age at seroconversion as the age at which at least one of the diabetes-associated autoantibodies was positive for the first time. If the child was autoantibody positive at 3 months of age, autoantibodies were measured in the maternal serum. Children with maternal autoantibodies were considered autoantibody negative. The incidence of seroconversion in each time period was expressed per 100 person-years of follow-up of children confirmed as autoantibody negative at the start of the time period.

Only 1,320 children who became autoantibody positive during follow-up were included in these analyses. Autoantibody profiles were analysed in three subgroups: (1) nondiabetic children who seroconverted to autoantibody positivity but never presented with $\geq$ two autoantibody species in two consecutive blood samples, referred to as 'single autoantibody positive'; (2) clinically healthy children positive for $\geq$ two autoantibodies at least in two consecutive blood samples, referred to as 'multiple autoantibodypositive'; and (3) autoantibody-positive children who progressed to diabetes. Information of later progression to diabetes was obtained from hospital records for those children lost to follow-up.

Genetic screening HLA-conferred susceptibility to type 1 diabetes was analysed using cord blood as described [19-21]. Carriers of the $H L A-D Q B 1$ genotypes *02/*0302 and $* 0302 / x\left(x \neq * 02,{ }^{*} 0301\right.$ or $\left.* 0602\right)$ were selected for follow-up. Children with the genotype $* 02 / * 0302$ were categorised into the high-risk group ( $8 \%$ risk before age 15 years), and those with the $* 0302 / x$ genotypes to the moderate-risk group (3\% risk). Before May 1997, DQB1 alleles $* 0602$ and $* 0603$ were indistinguishable in the screening assay. Children with $* 0602-3$ were classified as low-risk individuals and were excluded from the follow-up. Boys born in Turku in 1998 or later with the genotype $D Q B 1 * 02 / y-D Q A 1 * 05 / z(\mathrm{y} \neq * 0301, * 0302, * 0602, * 0603$; $\mathrm{z} \neq * 0201)$, representing moderate risk, were also recruited for follow-up.

Autoantibody assays Autoantibodies were analysed as described [13]. Briefly, ICA levels were measured using a standard indirect immunofluorescence assay with a detection limit of 2.5 Juvenile Diabetes Foundation Units (JDFU)
[22]. GADA levels were analysed using a radioligand assay with a cut-off limit for positivity of 5.36 relative units (RU) [23]. IAA levels were measured using a modified radioligand assay $[24,25]$. The cut-off limit for positivity was 3.48 RU. IA-2A levels were quantified with a radiobinding assay, the cut-off limit being $0.43 \mathrm{RU}$ [26].

Statistics The data were analysed using SAS software version 9.2 (SAS Institute, Cary, NC, USA). Due to skewed distributions of the autoantibody values, the data were presented as medians and quartiles and analysed with nonparametric methods. Continuous variables were analysed with the Wilcoxon rank sum test or the Kruskall-Wallis test, and were Bonferroni-corrected when appropriate. CochranMantel-Haenszel $\chi^{2}$ tests were used for categorical variables. Longitudinal analyses of the log-transformed autoantibody levels were performed with repeated-measures analysis of covariance using age at seroconversion, genetic risk level and sex as covariates. Progression to diabetes was modelled with covariate-adjusted Cox regression.

A subgroup of study children took part in a placebocontrolled clinical trial testing the effect of nasally administered insulin treatment [27]. All main results were checked using those children who did not participate in the nasal insulin trial and those who discontinued the follow-up to rule out possible confounding effects. $p$ values lower than 0.05 were considered significant.

\section{Results}

Study children Between November 1994 and December 2007, 122,636 newborn infants were screened for HLAconferred susceptibility to type 1 diabetes. Of these, 11,689 with genetic susceptibility were enrolled in the DIPP study (Fig. 1a), and 7,165 were followed from birth to either diagnosis of diabetes or the end of December 2008. As screening and recruitment were ongoing, the age of the children in the study ranged from 12 months to 14 years at the end of the follow-up period (Fig. 1b).

This study focused on the appearance and fate of the diabetes-associated autoantibodies and on the progression to overt type 1 diabetes among the 1,320 children who seroconverted to autoantibody positivity during the followup (947 of these were non-diabetic and positive for a single autoantibody, 189 were healthy but positive for multiple autoantibodies and 184 progressed to diabetes). In addition, four children previously negative for all four autoantibodies progressed to type 1 diabetes (electronic supplementary material [ESM] Table 1). The proportions of boys and girls were similar in all study groups (ESM Table 2). The median follow-up time was 7.7 years (interquartile range [IQR] 7.5-7.9). 
a

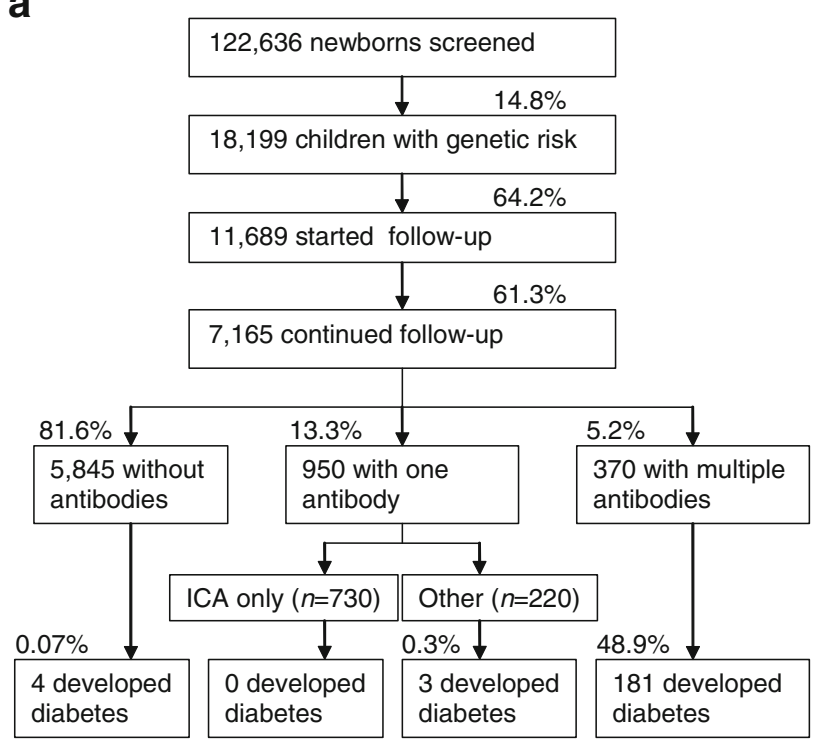

b

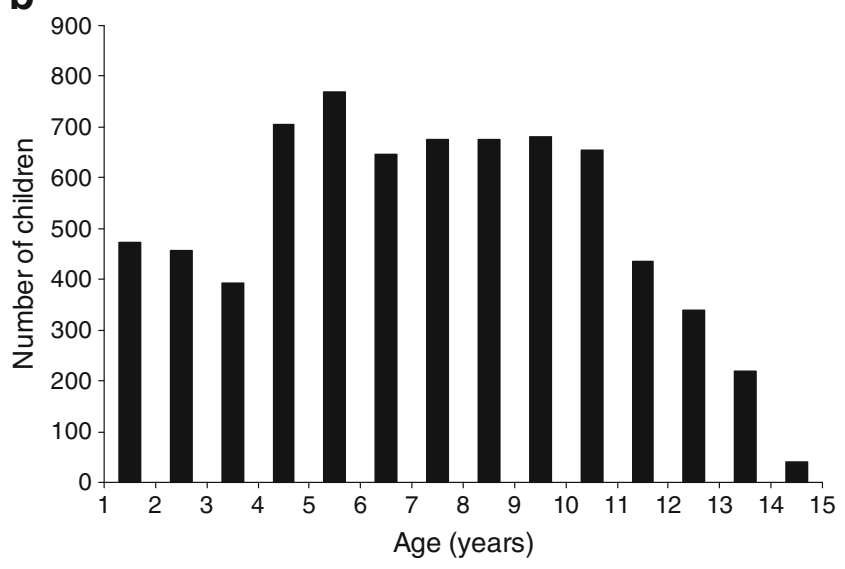

Fig. 1 Outline of the study (a) and the age distribution of the children participating in the follow-up, assessed in December 2008 (b). The percentages in a are the proportions with respect to the numbers in the boxes above (with the exception of $0.3 \%$, which is proportion of all 950 children with one antibody)

Seroconversion age Seroconversion to autoantibody positivity occurred at all ages (median at 3.1 years; Fig. 2a). Early seroconversion occurred more often among the children who progressed to diabetes during the follow-up than among the other children (ESM Table 2). Among the children who progressed to diabetes during the current followup, $118(64.1 \%)$ became autoantibody positive before the age of 2 years and $150(81.5 \%)$ before the age of 3 years (Fig. 2b). There was no difference in age at seroconversion between boys and girls.

A clear peak in the incidence of seroconversion to autoantibody positivity occurred during the second year of life (age 1.00-1.99), both when the very first autoantibody was considered (Fig. 2) and also when the appearances of IAA
(Fig. 3a), GADA (Fig. 3b) or IA-2A (Fig. 3c) were analysed separately. The 1 year peak in seroconversion rate was particularly conspicuous in children who later progressed to type 1 diabetes. Interestingly, seroconversion to positivity against three biochemically defined autoantigens occurred less frequently after the age of 4 years.

The seroconversion schedule was further examined by dividing the second year of life into 3 month periods. IAA, which was most commonly the first-emerging autoantibody species, most often appeared during the first quarter of the year (57 children, $40 \%$ of those who seroconverted during the second year), and less often during the last quarter (19 children). Other seroconversions occurred almost as often before and after the age of 18 months.

In contrast to other autoantibodies, seroconversion to ICA positivity (Fig. 3d) was quite uniformly distributed over the years of follow-up. Only a small peak was seen at the age of 1 year, and the drop in the seroconversion rate at 5 years was absent. Most of the children who developed

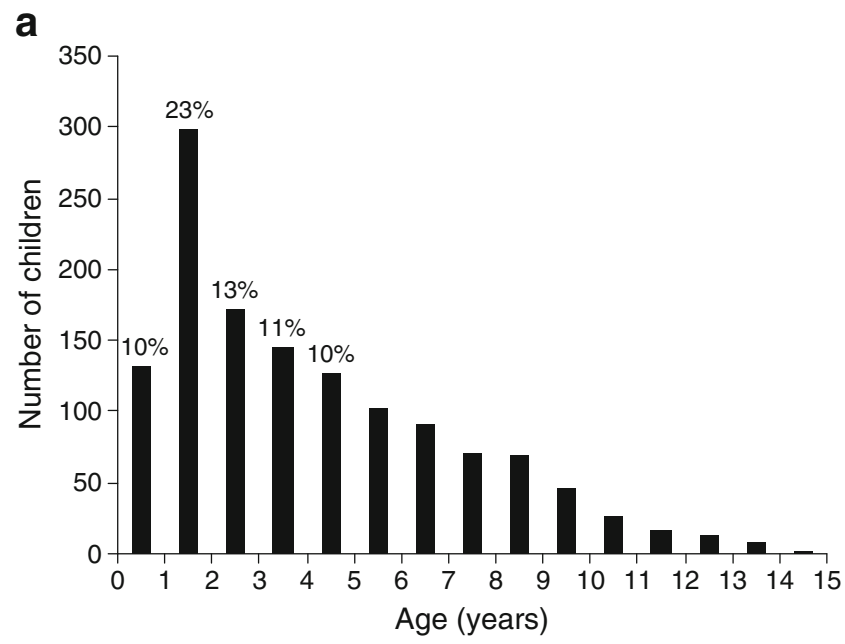

b

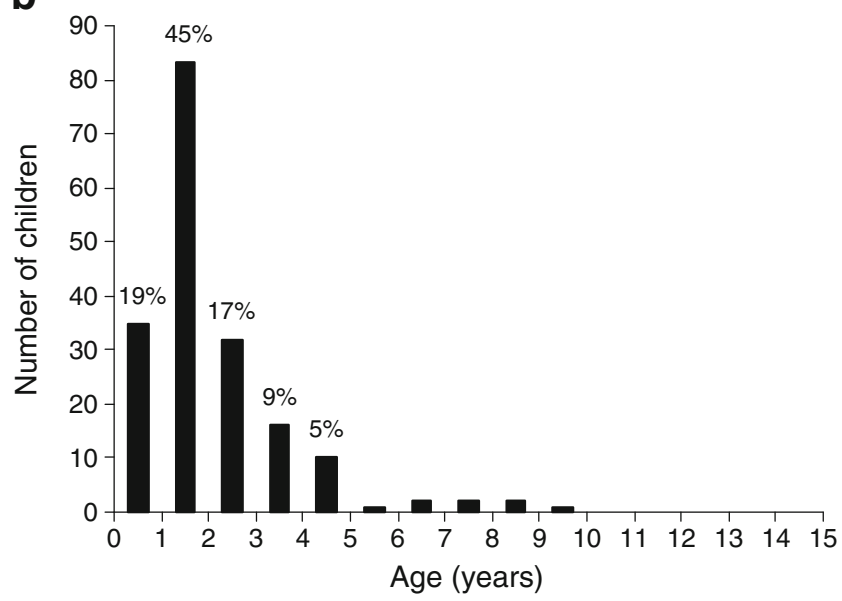

Fig. 2 Age at seroconversion in all autoantibody-positive children $(\mathbf{a} ; n=1,320)$, and in children who progressed to clinical diabetes $(\mathbf{b} ; n=184)$ 

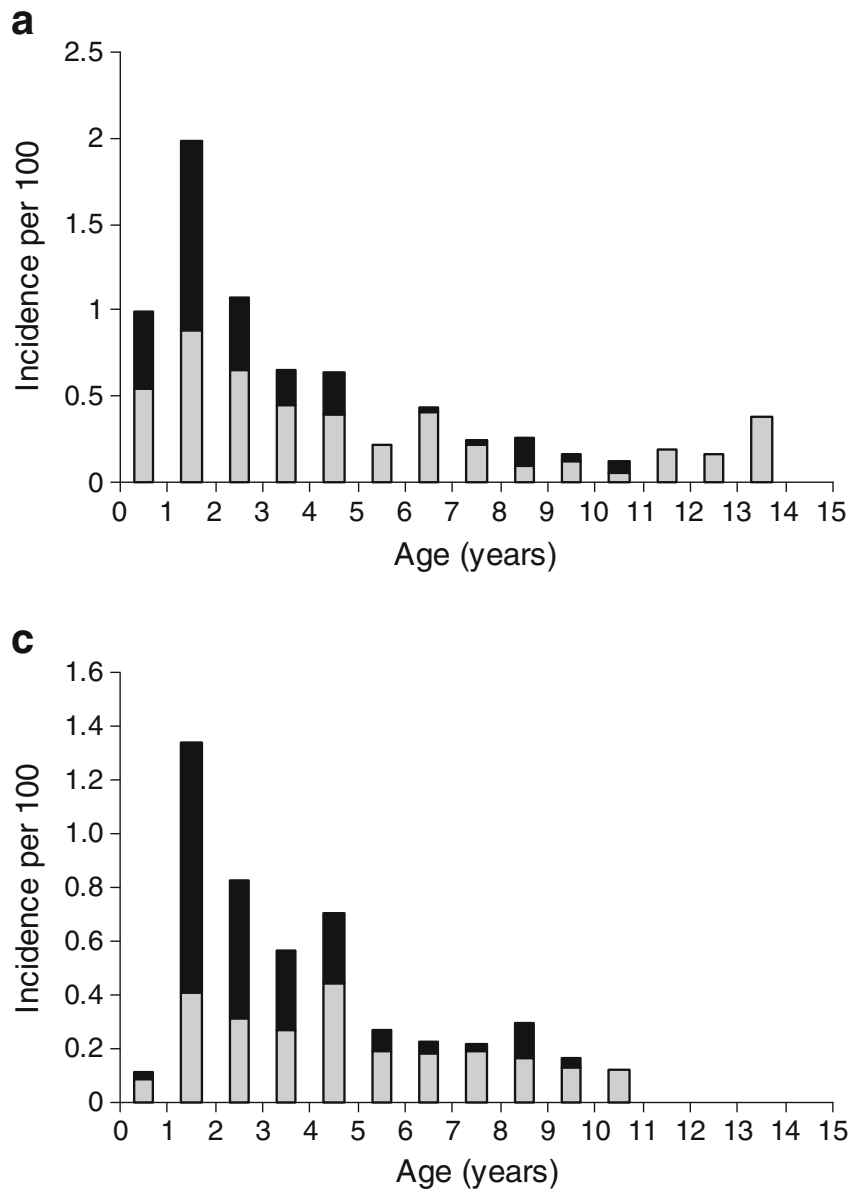

Fig. 3 Age at seroconversion to positivity for the diabetes-associated autoantibodies IAA (a), GADA (b), IA-2A (c) and ICA (d). Children who progressed to type 1 diabetes (black bars) and children who

autoantibodies at an age above 5 years became positive for ICA only, showed low autoantibody concentrations and did not show signs of progressive autoimmunity during the period preceding puberty.

Progression to multiple autoantibody positivity and diabetes At seroconversion, 1,103 (83.6\%) of the 1,320 autoantibody-positive children had only one type of autoantibody, and $931(84.4 \%)$ of these children have so far remained positive for only this single autoantibody. Sixtysix progressors $(36 \%$ of the 184 who developed diabetes during this study) and 106 children who developed multiple autoantibody positivity ( $56 \%$ of the 189 who later converted to positivity for multiple autoantibodies but did not progress to diabetes) presented first with only a single autoantibody.

The number of autoantibody species at the time of the first seroconversion showed a clear association with the frequency of sample collection $(p<0.0001)$. The children in the Turku subcohort were followed at 3 month intervals up to the age of 2 years, and subsequently at 6 month b

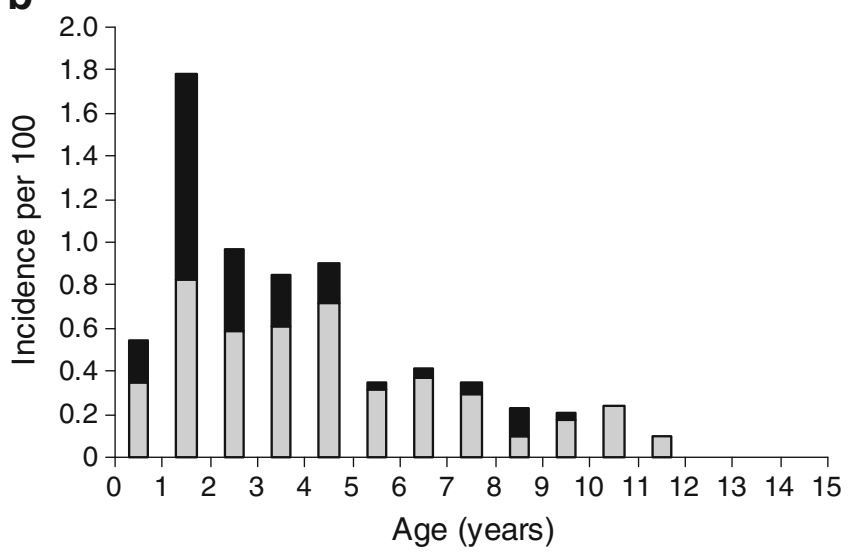

d

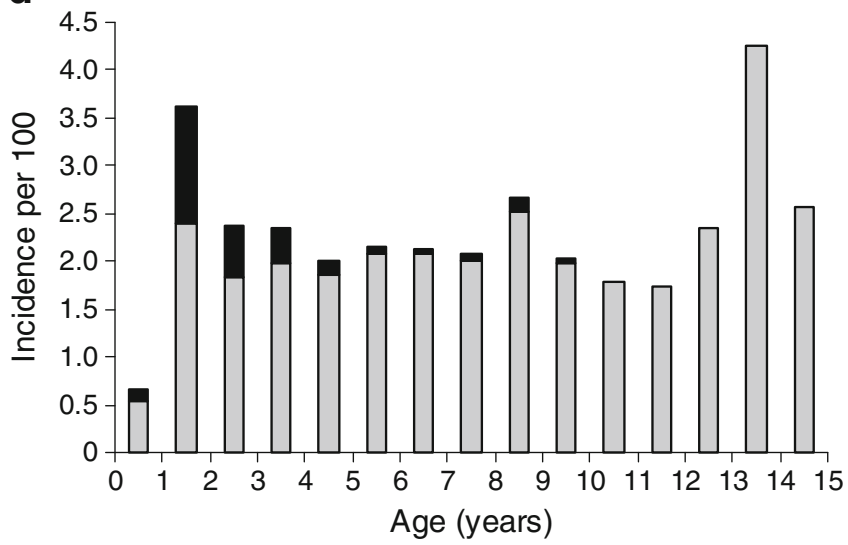

remained healthy (grey bars) are shown separately. The values have been calculated per 100 children in each age group to adjust for the age distribution due to variable length of follow-up

intervals, while samples in the Oulu and Tampere cohorts were first collected at the ages of 3 and 6 months, then biannually until the age of 2 years, and annually thereafter. Over half of those Turku children who later progressed to type 1 diabetes had only one autoantibody in the first seropositive sample, with only $14.1 \%$ having three or four autoantibodies; in contrast, $45.1 \%$ of the progressors in the cohort with longer blood-draw intervals had three or four autoantibodies in their first seropositive sample (ESM Fig. 1a). Most of the children who progressed to diabetes developed other autoantibodies during the follow-up, and $163(89 \%)$ children were positive for three or four autoantibodies before type 1 diabetes became manifest (ESM Fig. 1b).

The time from the emergence of the first autoantibody to multiple autoantibody positivity in children who later progressed to diabetes was short (median 0.00 years, IQR 0.00 0.28; in the Turku subcohort, 0.24 years, IQR $0.00-0.52$ ). The age at diagnosis of diabetes varied from 0.82 to 12.56 years, with a median of 4.28 years. Boys and girls 
showed no difference. The time from seroconversion to clinical diabetes varied from 0.02 to 12.3 years (median 2.47 years, $95 \%$ CI $0.18,7.13$ years). Progression to diabetes was clearly dependent on the duration of multipositivity, as each healthy year with autoantibodies decreased the probability of developing clinical diabetes before puberty by $30 \%$ (HR $0.70,95 \%$ CI $0.65,0.75 ; p<0.0001$ ).

Predictive role of different autoantibodies during followup Among the children who later progressed to clinical diabetes, IAA was most often the first autoantibody to appear (141 children, 77\%). Forty-four of these children presented with IAA alone, whereas 97 children had IAA together with one or more additional autoantibodies (Table 1). IA-2A was never the first autoantibody to appear in this series of children, and was rarely seen in the first autoantibody-positive sample with another autoantibody.

Children who progressed to clinical diabetes developed multiple autoantibodies during the follow-up. Only three children had only one antibody (IAA) before diagnosis; these three were all under 12 months of age at seroconversion and under 18 months at diagnosis, suggesting that the disease process was aggressive. By the time of diagnosis, these three children had two or three autoantibodies. Among all the children who progressed to diabetes, 177 (96\%) were or had been positive for ICA, 175 (95\%) for IAA, 148 (80\%) for IA$2 \mathrm{~A}$ and 146 (79\%) for GADA before diagnosis; these autoantibodies appeared in different combinations (Table 1). However, all 184 children were positive for either ICA or IAA, and 168 children (91\%) were positive for both. As described earlier, four children from the cohort progressed to type 1 diabetes in the absence of prior detection of autoantibodies (ESM Table 1).

Predictive value of early autoantibody concentration In the very first autoantibody-positive sample collected from each child, the concentration of each one of the four species of autoantibodies was slightly but significantly $(p<0.001)$ higher in children who later progressed to type 1 diabetes than in children who did not progress. The difference between the groups became more conspicuous during the following months, especially in the first 6 months after seroconversion. Antibody concentrations

Table 1 Autoantibody combinations in the first autoantibody-positive sample and clustered combinations of all autoantibodies detected in an individual at any time during the follow-up

\begin{tabular}{|c|c|c|c|c|c|c|}
\hline \multirow[t]{2}{*}{$\mathrm{AAb}$ profile } & \multicolumn{3}{|c|}{ First AAb-positive sample } & \multicolumn{3}{|c|}{ Any time during the follow-up } \\
\hline & $\begin{array}{l}\text { Single AAb, } \\
\text { no diabetes }\end{array}$ & $\begin{array}{l}\text { Multiple AAbs, } \\
\text { no diabetes }\end{array}$ & Diabetes & $\begin{array}{l}\text { Single AAb, } \\
\text { no diabetes }\end{array}$ & $\begin{array}{l}\text { Multiple AAbs, } \\
\text { no diabetes }\end{array}$ & Diabetes \\
\hline IAA only & $79(8.3)$ & $44(23.3)$ & $44(23.9)$ & $47(5.0)$ & $0(0.0)$ & $3(1.6)$ \\
\hline GADA only & $73(7.7)$ & $32(16.9)$ & $13(7.1)$ & $44(4.6)$ & $0(0.0)$ & $0(0.0)$ \\
\hline ICA only & $762(80.5)$ & $27(14.3)$ & $9(4.9)$ & $730(77.1)$ & $0(0.0)$ & $0(0.0)$ \\
\hline IA-2A only & $17(1.8)$ & $3(1.6)$ & $0(0.0)$ & $8(0.8)$ & $0(0.0)$ & $0(0.0)$ \\
\hline $\mathrm{IAA}+\mathrm{ICA}$ & $5(0.5)$ & $19(10.1)$ & $25(13.6)$ & $47(5.0)$ & $23(12.2)$ & $10(5.4)$ \\
\hline $\mathrm{IAA}+\mathrm{GADA}$ & $2(0.2)$ & $10(5.3)$ & $16(8.7)$ & $2(0.2)$ & $0(0.0)$ & $3(1.6)$ \\
\hline $\mathrm{IAA}+\mathrm{IA}-2 \mathrm{~A}$ & $0(0.0)$ & $0(0.0)$ & $2(1.1)$ & $0(0.0)$ & $0(0.0)$ & $1(0.5)$ \\
\hline GADA+ICA & $4(0.4)$ & $18(9.5)$ & $9(4.9)$ & $43(4.5)$ & $34(18.0)$ & $2(1.1)$ \\
\hline GADA+IA-2A & $0(0.0)$ & $1(0.5)$ & $0(0.0)$ & $0(0.0)$ & $0(0.0)$ & $0(0.0)$ \\
\hline $\mathrm{ICA}+\mathrm{IA}-2 \mathrm{~A}$ & $1(0.1)$ & $6(3.2)$ & $5(2.7)$ & $12(1.3)$ & $5(2.6)$ & $2(1.1)$ \\
\hline $\mathrm{IAA}+\mathrm{GADA}+\mathrm{ICA}$ & $4(0.4)$ & $15(7.9)$ & $19(10.3)$ & $12(1.3)$ & $20(10.6)$ & $18(9.8)$ \\
\hline $\mathrm{IAA}+\mathrm{GADA}+\mathrm{IA}-2 \mathrm{~A}$ & $0(0.0)$ & $0(0.0)$ & $1(0.5)$ & $0(0.0)$ & $0(0.0)$ & $0(0.0)$ \\
\hline $\mathrm{IAA}+\mathrm{ICA}+\mathrm{IA}-2 \mathrm{~A}$ & $0(0.0)$ & $3(1.6)$ & $11(6.0)$ & $1(0.1)$ & $5(2.6)$ & $22(12.0)$ \\
\hline $\mathrm{GADA}+\mathrm{ICA}+\mathrm{IA}-2 \mathrm{~A}$ & $0(0.0)$ & $6(3.2)$ & $7(3.8)$ & $1(0.1)$ & $14(7.4)$ & $5(2.7)$ \\
\hline All four AAbs & $0(0.0)$ & $5(2.6)$ & $23(12.5)$ & $0(0.0)$ & $88(46.6)$ & $118(64.1)$ \\
\hline Total & 947 & 189 & 184 & 947 & 189 & 184 \\
\hline
\end{tabular}

Data are presented as $n(\%)$

The number and proportion of individuals with various antibody profiles with either single or multiple autoantibodies or overt type I diabetes during the study are shown. Multiple autoantibody positivity was defined as having at least two autoantibodies simultaneously in at least two consecutive samples. Autoantibodies are not necessarily persistent; thus, autoantibodies given in the clustered combinations may have been detected in separate samples

AAb, autoantibody 
in children who were positive for a single autoantibody remained low or declined, whereas concentrations in children who later progressed to diabetes rose quickly. In children who were positive for multiple autoantibodies but who did not progress to diabetes, the increase in antibody concentrations was less clear, or absent in the case of IAA (Fig. 4a). These differences in the profiles of the autoantibody titres persisted, and for IA-2A the difference became even more pronounced over the first 1.5 years after seroconversion (Fig. 4b).

As IAA was typically the first autoantibody to appear, IAA values were studied more closely around the time of seroconversion and thereafter. Antibody concentrations
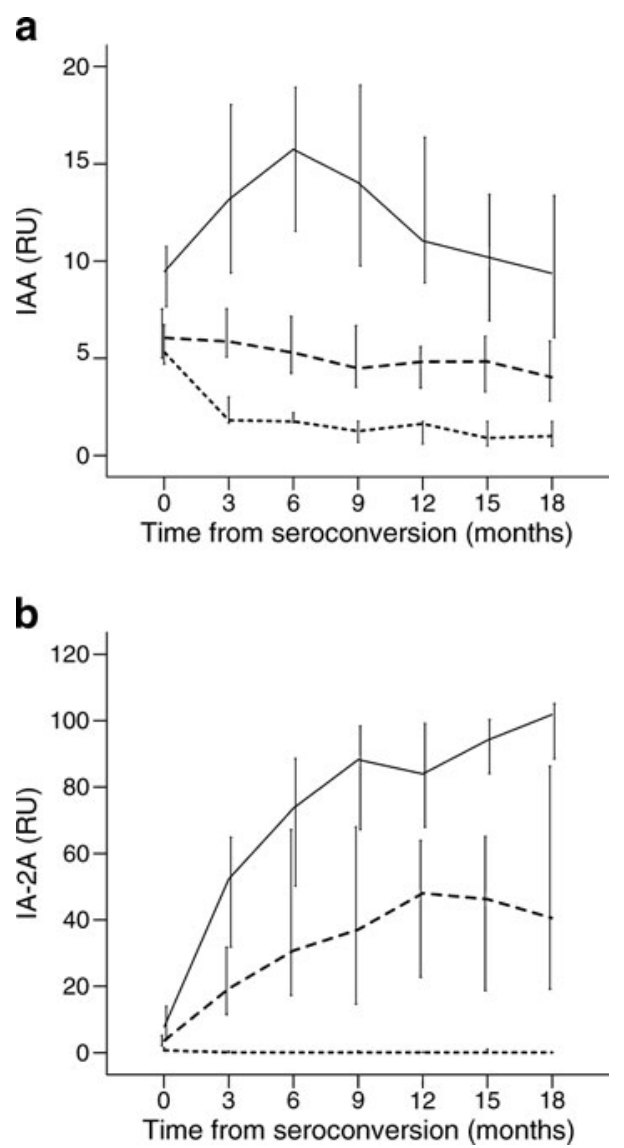

Fig. 4 Median autoantibody levels in non-diabetic singleautoantibody-positive children (dotted line), non-diabetic multipleautoantibody-positive children (dashed line) and progressors to type 1 diabetes (straight line) at seroconversion and at 3, 6, 9, 1215 and 18 months post-seroconversion. Levels of IAA, typically the first autoantibody to appear (a) and IA-2A, the last to appear (b) are presented. The values differed significantly at different time points $(p<0.001$ for IAA and 0.0013 for IA-2A) and between the groups ( $p<0.001$ for both IAA and IA-2A). During the follow-up the profile of values in various groups was also different $(p<0.001$ for both IAA and IA-2A). The results remained similar when adjusted for the following covariates: sex, genetic risk and age at seroconversion. Error bars show $95 \% \mathrm{CI}$ were already predictive of progression to diabetes in the first IAA-positive sample ( $p<0.0001$; Fig. 5a). Progression to diabetes was fastest in children whose IAA value was in the highest quartile. The prognostic value of IAA level was even more apparent 6 months after seroconversion to IAA positivity $(p<0.0001$; Fig. $5 b)$.

\section{Discussion}

This study shows that children with HLA-conferred susceptibility to type 1 diabetes who progressed to clinical diabetes before puberty developed diabetesassociated autoantibodies predominantly before 4 years of age, with a peak in seroconversion rate during the second year of life. An increase in autoantibody concentrations during the next few months following initial seroconversion efficiently discriminated children who progressed early to clinical diabetes from those who remained non-diabetic.

The strength of this study is the high number of study children, including 188 children with short intervals between visits, who progressed to diabetes. This made it possible to determine more exactly the timing of seroconversion than was possible in earlier studies. One of the study limitations was using ICA as the primary screening tool for autoimmunity during the first study years. It meant that some children positive for a single autoantibody (other than ICA) or two or more biochemically defined autoantibodies in the absence of ICA were likely to have been missed. However, permanent positivity for other autoantibodies was only rarely seen in children who were negative for ICA. ICA as used here is not particularly good for screening as it picks up a large number of children who never develop other autoantibodies or progress to diabetes. Use of a higher limit for positivity would diminish the number of autoantibody-positive children without influencing the detection of those children who later progress to diabetes. Analysis of children who discontinued did not change interpretation of the data and all results remained practically similar even after the Nasal Insulin Trial children were excluded from the analyses (data not shown).

The BABYDIAB study was the first to suggest that seroconversions to autoantibody positivity are common before the age of 2 years [28]. However, the study was restricted to offspring of parents affected by type 1 diabetes, and the samples were drawn at the ages of 9, 24 and 60 months. By including in our study a subcohort with a more frequent monitoring schedule, we demonstrated that potentially important findings may remain hidden even with biannual sampling during the first years of life. Our data indicate that in a setting where samples are drawn from the children at 3 month intervals starting at birth, a single autoantibody species usually appears first, followed within 1 year (90th percentile, 0.99 year) by one or more additional autoantibody species in children 
Fig. 5 Diabetes-free survival curves during follow-up. Children whose IAA values were in the first, second, third or fourth value quartiles were compared. The lowest $25 \%$ (straight black line), lower middle $25 \%$ (dotted black line), upper middle $25 \%$ (straight grey line) and highest $25 \%$ (dotted grey line) are shown. The IAA values were studied in the first IAA-positive sample (a) and 6 months later (b). At both time points $(\mathbf{a}, \mathbf{b})$ the groups differed significantly from each other $(p<0.0001)$. The cut-off limits for quartiles were $4.45,6.74$ and 13.84 RU at the time of seroconversion and 2.07, 5.45 and 17.15 RU 6 months later. In a there was no difference in progression to diabetes between the lowest two quartiles. In all other comparisons the risk of the disease increased with increasing IAA level ( $p=0.006$ for two vs three quartiles and $p<0.001$ for all others). In $\mathbf{b}$ the risk of diabetes was higher in the highest quartile compared with all other IAA levels $(p<0.001$ for all comparisons)
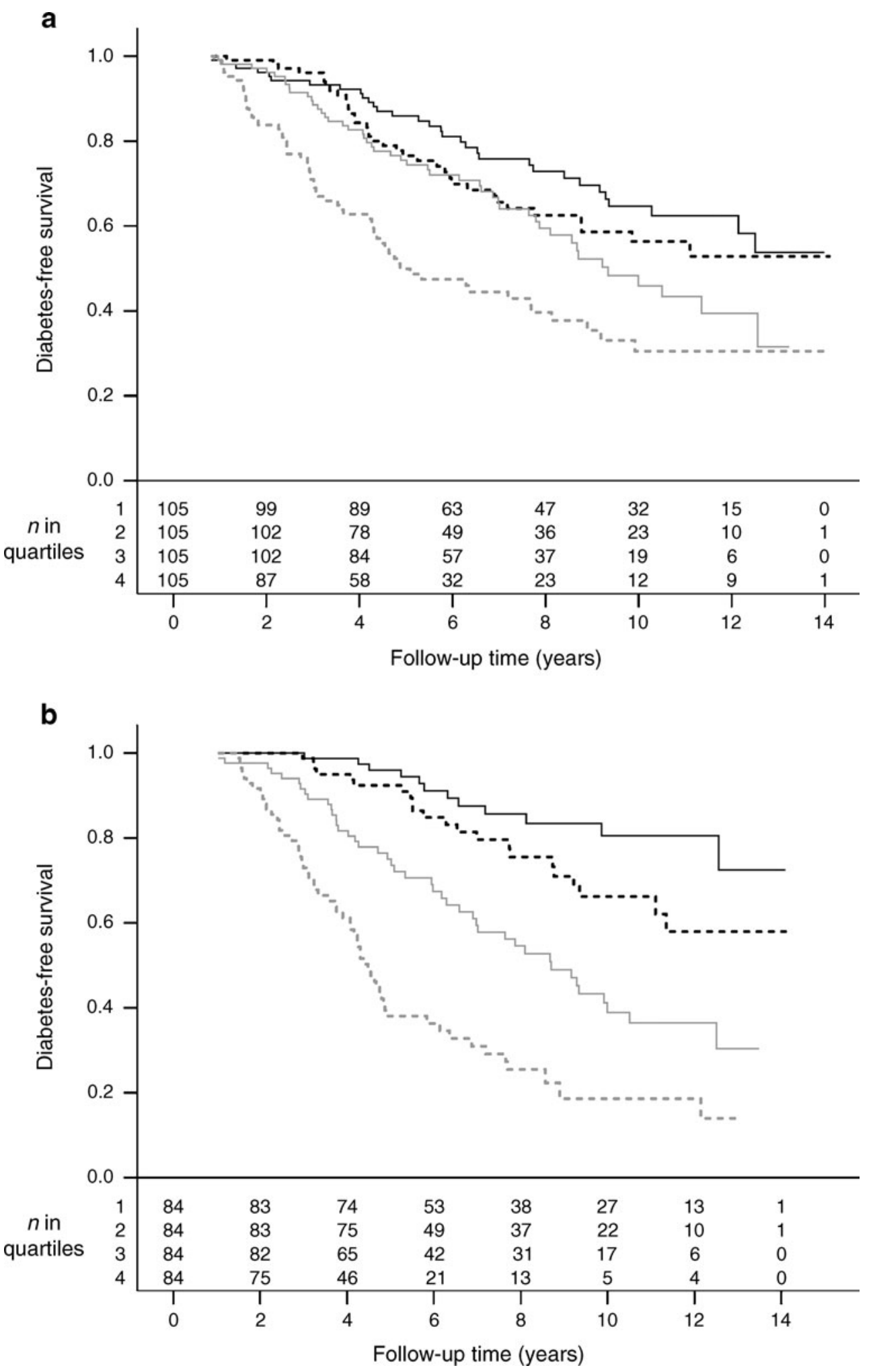

who progress to diabetes before puberty. More frequent blood draws are justified to pinpoint more closely the moment of seroconversion, which is probably important for the detection of the environmental triggers of autoimmunity, and for preventive interventions in the future. Thus, we recommend a 3 month sampling interval during the first years of life in studies aimed at identifying exogenous triggers of beta cell autoimmunity. This approach has been adopted in, for 
example, The Environmental Determinants of Diabetes in the Young study [29].

The peak in the seroconversion rate was noted at a surprisingly young age, with a median at around 1 year. The finding that the seroconversion rate of IAA was higher at the age of 12-17 months compared with the rate at the age of 18-23 months strongly suggests that early seroconversions are not random phenomena but emphasise the importance of early environmental events in the pathogenesis of beta cell autoimmunity. Whether these factors are dietary or associated with infection or something else remains open. In any case, it is highly likely that the increase in diabetes incidence before puberty and particularly among children under the age of 5 years [30] is due to processes that, in most cases, begin during the first 2 years of life, or even during pregnancy. Previous $[13,28]$ and our data (not shown) further show that children who seroconvert before 2 years are more prone to progress to clinical type 1 diabetes before puberty than children who seroconvert at an older age.

The majority of the autoantibody-positive children were persistently positive for only one autoantibody, had low autoantibody levels and carried a low risk of progression to clinical diabetes. In contrast, children with multiple autoantibodies were at a substantially higher risk of progressing to clinical disease. They resembled children who progressed to diabetes during the follow-up, but they were older at seroconversion, progressed to multiple antibody positivity more slowly and had lower concentrations of autoantibodies in the samples collected soon after seroconversion. This indicates that they had a less aggressive diabetic disease process, but a majority of them will probably present with clinical type 1 diabetes at a later age. A recent Finnish survey showed that all children who were recruited from the general population and tested positive for GADA and IA-2A at a mean age of 11.8 years progressed to overt type 1 diabetes over the next 21 years [31]. The time interval between the emergence of the first autoantibody and clinical diabetes varied in the current study from a few months to several years, even in children who were very young at seroconversion. For example, two girls who seroconverted to IAA positivity at the age of 9 months and carried the same high-risk $H L A-D Q B 1$ genotype developed clinical diabetes at the ages of 12 months and 7.7 years. The screening procedure, however, only defined the $H L A-D Q B 1$ locus with the strongest disease risk association and did not take into account class I HLA loci recently shown to be important for progression of the autoimmune process to clinical disease [32, 33] or multiple loci outside the HLA region [34]. The reasons behind such variation in the progression rate remain open, and predictive models for narrowing the estimations of the duration of the prediabetic period are needed.

Similar to children positive for multiple autoantibodies [16, 17] children with high ICA titres are at high risk of progressing to diabetes $[35,36]$, as high ICA levels are strongly associated with positivity for multiple autoantibodies. Children with high concentrations of IAA and IA-2A have also been observed to be at increased risk of diabetes [15, 37], and future progressors show higher concentrations of IAA in the first positive sample than other autoantibody-positive children [38]. In contrast, GADA concentrations did not differ between those who progressed to diabetes and those who did not [15, 37]. Sosenko et al [39] have demonstrated decreased GADA and increased IA-2A titres from baseline to diagnosis. This is in agreement with our data showing an increase in IA-2A levels during the first months after seroconversion. A recent study [40] has shown that initial IAA levels predict the age of diabetes diagnosis, which is in agreement with our data. Our data further show that the predictive value of IAA level is even better 6 months after seroconversion. Although showing a statistically significant difference, the autoantibody concentrations at seroconversion were quite similar in all children in the present study. The differences between the progressors and non-progressors increased conspicuously soon after seroconversion. These data suggest that autoantibody values during the first year after seroconversion are more informative than single values measured in samples collected at random time points, e.g. during screening of populations of schoolchildren.

In conclusion, all but four children in our large DIPP cohort seroconverted to autoantibody positivity before presenting with clinical type 1 diabetes. There was a pronounced peak in seroconversion during the second year of life, indicating that triggers of diabetes-associated autoimmunity should be particularly sought in this age group or earlier. Autoantibody concentrations increased markedly 318 months after seroconversion in children who progressed to diabetes before puberty, indicating that not only autoantibody positivity but also autoantibody concentrations are of importance in predicting risk of clinical type 1 diabetes. Future studies are needed to find the mechanisms underlying this early and aggressive start in the autoimmune process that leads to clinical diabetes.

Acknowledgements All families participating in the DIPP study are warmly acknowledged for their valuable contribution. The authors also thank the personnel in the study clinics and in the genetic and autoantibody laboratories for their significant contribution to the success of the DIPP study.

Funding This study was supported by the Academy of Finland (grants 68292 and 114666), Juvenile Diabetes Research Foundation International (grants 4-1998-274, 4-1999-731, 4-2001-435), the TEKES National Technology Agency of Finland, the Finnish Office for Health Technology Assessment, the Päivikki and Sakari Sohlberg Foundation, the Sigrid Juselius Foundation, the Signe and Ane Gyllenberg Foundation, the Varsinais-Suomi Regional Fund of the Finnish Cultural Foundation, the European Union (grant BMH4-CT98-3314), the Special Research Funds for University Hospitals in Finland, the Diabetes Research Foundation, Finland, the Emil Aaltonen Foundation and the Foundation for Pediatric Research, Finland. 
Contribution statement VP, KN-S, MS and OS analysed data and drafted the manuscript. OS, TS, JI, MK, RV and HH were responsible for the conception of the study. All authors participated in revising the manuscript and approved the final version.

Duality of interest The authors declare that there is no duality of interest associated with this manuscript.

\section{References}

1. Onkamo P, Väänänen S, Karvonen M, Tuomilehto J (1999) Worldwide increase in incidence of type I diabetes - the analysis of the data on published incidence trends. Diabetologia 42:1395-1403

2. DIAMOND Project Group (2006) Incidence and trends of childhood type 1 diabetes worldwide 1990-1999. Diabet Med 23:857866

3. Patterson CC, Dahlquist GG, Gyürüs E, Green A, Soltész G, EURODIAB Study Group (2009) Incidence trends for childhood type 1 diabetes in Europe during 1989-2003 and predicted new cases 2005-2020: a multicentre prospective registration study. Lancet 373:2027-2033

4. Pitkäniemi J, Onkamo P, Tuomilehto J, Arjas E (2004) Increasing incidence of type 1 diabetes - role for genes? BMC Genet 5:5

5. Harjutsalo V, Sjöberg L, Tuomilehto J (2008) Time trends in the incidence of type 1 diabetes in Finnish children: a cohort study. Lancet 371:1777-1782

6. Gardner SG, Bingley PJ, Sawtell PA, Weeks S, Gale EA (1997) Rising incidence of insulin dependent diabetes in children aged under 5 years in the Oxford region: time trend analysis. The Bart'sOxford Study Group. BMJ 315:713-717

7. Todd JA (1995) Genetic analysis of type 1 diabetes using whole genome approaches. Proc Natl Acad Sci U S A 92:8560-8565

8. Ilonen J, Sjöroos M, Knip M et al (2002) Estimation of genetic risk for type 1 diabetes. Am J Med Genet 115:30-36

9. Gillespie KM, Bain SC, Barnett AH et al (2004) The rising incidence of childhood type 1 diabetes and reduced contribution of high-risk HLA haplotypes. Lancet 364:1699-1700

10. Knip M, Veijola R, Virtanen SM, Hyöty H, Vaarala O, Åkerblom HK (2005) Environmental triggers and determinants of type 1 diabetes. Diabetes 54:S125-S136

11. Oresic M, Simell S, Sysi-Aho M et al (2008) Dysregulation of lipid and amino acid metabolism precedes islet autoimmunity in children who later progress to type 1 diabetes. J Exp Med 205:29752984

12. Notkins AL, Lernmark A (2001) Autoimmune type 1 diabetes: resolved and unresolved issues. J Clin Invest 108:1247-1252

13. Siljander HT, Simell S, Hekkala A et al (2009) Predictive characteristics of diabetes-associated autoantibodies among children with HLA-conferred disease susceptibility in the general population. Diabetes 58:2835-2842

14. Lambert AP, Gillespie KM, Thomson G et al (2004) Absolute risk of childhood-onset type 1 diabetes defined by human leukocyte antigen class II genotype: a population-based study in the United Kingdom. J Clin Endocrinol Metab 89:4037-4043

15. Kulmala P, Savola K, Petersen JS et al (1998) Prediction of insulindependent diabetes mellitus in siblings of children with diabetes. A population-based study. The Childhood Diabetes in Finland Study Group. J Clin Invest 101:327-336

16. Verge CF, Gianani R, Kawasaki E et al (1996) Prediction of type I diabetes in first-degree relatives using a combination of insulin, GAD, and ICA512bdc/IA-2 autoantibodies. Diabetes 45:926-933
17. Bingley PJ, Christie MR, Bonifacio E et al (1994) Combined analysis of autoantibodies improves prediction of IDDM in islet cell antibody-positive relatives. Diabetes 43:1304-1310

18. Keskinen P, Korhonen S, Kupila A et al (2002) First-phase insulin response in young healthy children at genetic and immunological risk for type I diabetes. Diabetologia 45:1639-1648

19. Sjöroos M, Iitiä A, Ilonen J, Reijonen H, Lövgren T (1995) Triplelabel hybridization assay for type-1 diabetes-related HLA alleles. Biotechniques 18:870-877

20. Ilonen J, Reijonen H, Herva E et al (1996) Rapid HLA-DQB1 genotyping for four alleles in the assessment of risk for IDDM in the Finnish population. The Childhood Diabetes in Finland (DiMe) Study Group. Diabetes Care 19:795-800

21. Nejentsev S, Sjöroos M, Soukka T et al (1999) Population-based genetic screening for the estimation of type 1 diabetes mellitus risk in Finland: selective genotyping of markers in the HLA-DQB1, HLA-DQA1 and HLA-DRB1 loci. Diabet Med 16:985-992

22. Bottazzo GF, Florin-Christensen A, Doniach D (1974) Islet-cell antibodies in diabetes mellitus with autoimmune polyendocrine deficiencies. Lancet 2:1279-1283

23. Savola K, Sabbah E, Kulmala P, Vähäsalo P, Ilonen J, Knip M (1998) Autoantibodies associated with type I diabetes mellitus persist after diagnosis in children. Diabetologia 41:1293-1297

24. Williams AJ, Bingley PJ, Bonifacio E, Palmer JP, Gale EA (1997) A novel micro-assay for insulin autoantibodies. J Autoimmun 10:473-478

25. Ronkainen MS, Hämäläinen AM, Koskela P, Åkerblom HK, Knip M, Finnish Trigr Study Group (2001) Pregnancy induces nonimmunoglobulin insulin-binding activity in both maternal and cord blood serum. Clin Exp Immunol 124:190-196

26. Savola K, Bonifacio E, Sabbah E et al (1998) IA-2 antibodies-a sensitive marker of IDDM with clinical onset in childhood and adolescence. Childhood Diabetes in Finland Study Group. Diabetologia 41:424-429

27. Näntö-Salonen K, Kupila A, Simell S et al (2008) Nasal insulin to prevent type 1 diabetes in children with HLA genotypes and autoantibodies conferring increased risk of disease: a doubleblind, randomised controlled trial. Lancet 372:1746-1755

28. Ziegler AG, Hummel M, Schenker M, Bonifacio E (1999) Autoantibody appearance and risk for development of childhood diabetes in offspring of parents with type 1 diabetes: the 2-year analysis of the German BABYDIAB Study. Diabetes 48:460-468

29. TEDDY Study Group (2008) The Environmental Determinants of Diabetes in the Young (TEDDY) study. Ann N Y Acad Sci 1150:1-13

30. Karvonen M, Pitkäniemi J, Tuomilehto J (1999) The onset age of type 1 diabetes in Finnish children has become younger. The Finnish Childhood Diabetes Registry Group. Diabetes Care 22:1066-1070

31. Knip M, Korhonen S, Kulmala P et al (2010) Prediction of type 1 diabetes in the general population. Diabetes Care 33:1206-1212

32. Nejentsev S, Howson JM, Walker NM et al (2007) Localization of type 1 diabetes susceptibility to the MHC class I genes HLA-B and HLA-A. Nature 450:887-892

33. Lipponen K, Gombos Z, Kiviniemi M et al (2010) Effect of HLA class I and class II alleles on progression from autoantibody positivity to overt type 1 diabetes in children with risk-associated class II genotypes. Diabetes 59:3253-3256

34. Barrett JC, Clayton DG, Concannon P et al (2009) Genome-wide association study and meta-analysis find that over 40 loci affect risk of type 1 diabetes. Nat Genet 41:703-707

35. Bonifacio E, Bingley PJ, Shattock M et al (1990) Quantification of islet-cell antibodies and prediction of insulin-dependent diabetes. Lancet 335:147-149

36. Thivolet $\mathrm{C}$, Beaufrère B, Geburher L, Chatelain P, Orgiazzi J, François R (1991) Autoantibodies and genetic factors associated with the 
development of type 1 (insulin-dependent) diabetes mellitus in first degree relatives of diabetic patients. Diabetologia 34:186-191

37. Achenbach P, Warncke K, Reiter J et al (2004) Stratification of type 1 diabetes risk on the basis of islet autoantibody characteristics. Diabetes 53:384-392

38. Barker JM, Barriga KJ, Yu L et al (2004) Prediction of autoantibody positivity and progression to type 1 diabetes: Diabetes Autoimmunity Study in the Young (DAISY). J Clin Endocrinol Metab 89:3896-3902
39. Sosenko JM, Skyler JS, Palmer JP et al (2011) A longitudinal study of GAD65 and ICA512 autoantibodies during the progression to type 1 diabetes in Diabetes Prevention Trial-type 1 (DPT-1) Participants. Diabetes Care 34:2435-2437

40. Steck AK, Johnson K, Barriga KJ et al (2011) Age of islet autoantibody appearance and mean levels of insulin, but not GAD or IA-2 autoantibodies, predict age of diagnosis of type 1 diabetes: diabetes autoimmunity study in the young. Diabetes Care 34:1397-1399 